\title{
Mechanical Autonomous Stochastic Heat Engine
}

\author{
Marc Serra-Garcia, ${ }^{1, *}$ André Foehr, ${ }^{1}$ Miguel Molerón, ${ }^{1}$ Joseph Lydon, ${ }^{1}$ Christopher Chong, ${ }^{2}$ and Chiara Daraio ${ }^{1,3}$ \\ ${ }^{1}$ Department of Mechanical and Process Engineering, Swiss Federal Institute of Technology (ETH), 8092 Zürich, Switzerland \\ ${ }^{2}$ Department of Mathematics, Bowdoin College, Brunswick, Maine 04011, USA \\ ${ }^{3}$ Engineering and Applied Science, California Institute of Technology, Pasadena, California 91125, USA
}

(Received 1 February 2016; published 28 June 2016)

\begin{abstract}
Stochastic heat engines are devices that generate work from random thermal motion using a small number of highly fluctuating degrees of freedom. Proposals for such devices have existed for more than a century and include the Maxwell demon and the Feynman ratchet. Only recently have they been demonstrated experimentally, using, e.g., thermal cycles implemented in optical traps. However, recent experimental demonstrations of classical stochastic heat engines are nonautonomous, since they require an external control system that prescribes a heating and cooling cycle and consume more energy than they produce. We present a heat engine consisting of three coupled mechanical resonators (two ribbons and a cantilever) subject to a stochastic drive. The engine uses geometric nonlinearities in the resonating ribbons to autonomously convert a random excitation into a low-entropy, nonpassive oscillation of the cantilever. The engine presents the anomalous heat transport property of negative thermal conductivity, consisting in the ability to passively transfer energy from a cold reservoir to a hot reservoir.
\end{abstract}

DOI: 10.1103/PhysRevLett.117.010602

Thermodynamics in low-dimensional systems far from equilibrium is not well understood, to the point that essential quantities such as work [1] or entropy [2] do not have universally valid definitions in such systems. Formulating a physical theory for thermal processes in low-dimensional systems is the subject of stochastic thermodynamics [3], an emergent field that has resulted in the discovery of a variety of microscopic heat engines [4-12] and fluctuation theorems [13-16], and has provided new insights on the connection between information and energy [5,17-20]. A central problem in stochastic thermodynamics is the construction and analysis of stochastic heat engines, the low-dimensional analogs of conventional thermal machines. A stochastic heat engine is a low-dimensional device that operates between two thermal baths at different temperatures, and is able to produce work while suppressing the randomness inherent in thermal motion [1,4-7,21-23]. Thermal engine operation is characterized by the presence of nonpassive states of motion, which have lower entropy (for the same energy) than equilibrium states $[24,25]$ and therefore allow the extraction of energy without an associated entropy flow [1]. The interest in stochastic heat machines is motivated by the desire to understand energy conversion processes at the fundamental level. This understanding, coupled with modern nanofabrication techniques, is expected to result in more efficient and powerful thermal machines.

The concept of the stochastic heat engine dates back to the classical thought experiments of the Maxwell demon $[18,26]$ and the Feynman ratchet [27,28]. Only very recently have working experimental realizations of the stochastic heat engine been reported on [4-8]. The bulk of these experimental realizations is based on the manipulation of a particle in an optical trap, and include the implementation of adiabatic processes [7], feedback loops [5], as well as Stirling [6] and Carnot [4] cycles. These engines are nonautonomous since they operate under externally prescribed cycles. As a consequence, the energy they require to operate is orders of magnitude higher than the work they produce, and the externally prescribed dynamical cycle masks the significant challenges that hinder the description of autonomous physical systems [1]. In this Letter, we describe a classical mechanical system that realizes autonomous thermal engine operation. Our proposed engine consists of two coupled ribbons and a cantilever beam connected to one of the ribbons. The presence of nonlinearity in the ribbons dynamically and autonomously adjusts the coupling to the hot and cold thermal baths, thus replacing the external control unit in the nonautonomous realizations reported until now. We demonstrate this concept in a macroscopic tabletop setup, utilizing two $30 \mathrm{~cm}$ long brass ribbons and a $40.6 \mathrm{~cm}$ long steel cantilever [29]. Since our system is too large to exhibit sufficient Brownian motion at room temperature, we magnetically excite one of the ribbons using white noise to simulate a high effective temperature, $T_{H}$ (up to $5 \times 10^{18} \mathrm{~K}$ ). In the Supplemental Material [29], we numerically demonstrate the engine's scaling to microscopic dimensions, where Brownian motion at experimentally accessible temperatures $\left(150^{\circ} \mathrm{C}\right)$ is sufficient to induce measurable self-sustained thermal engine operation.

The thermal cycle of our engine is analogous to the classical Stirling cycle [Figs. 1(a) and 1(b)], which consists of four steps performed on a working fluid-heating, expansion, cooling, and compression. The ribbon attached to the cantilever (main ribbon, with displacement denoted $x_{M}$ ) plays the role of the working fluid. This ribbon is in 

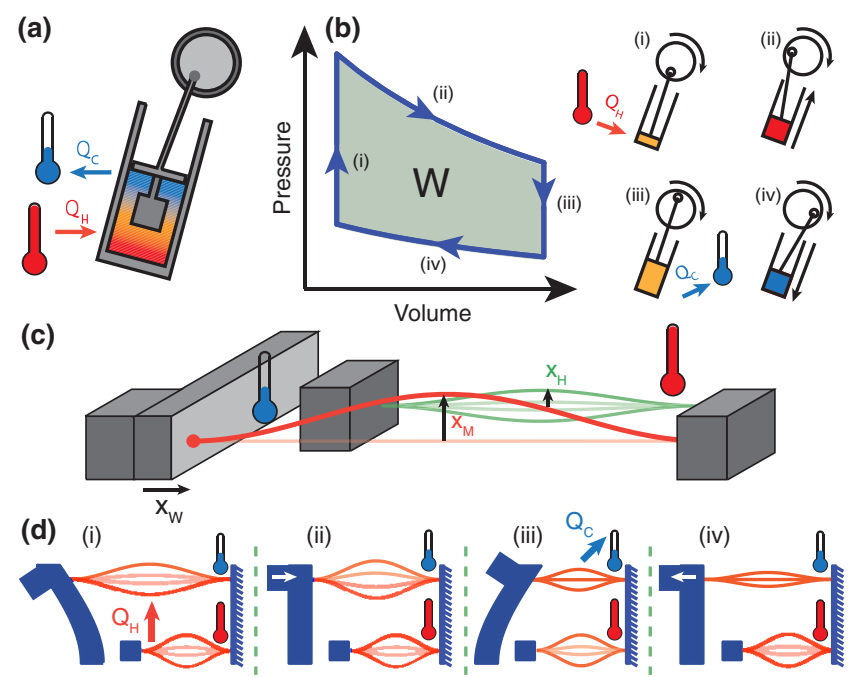

FIG. 1. Cyclic thermal engines. (a) Stirling heat engine. The engine uses a piston to cyclically compress and expand a gas. A secondary piston displaces the gas and regulates the coupling to the hot and cold reservoirs. (b) Thermal cycle for the Stirling engine. The difference in pressure during expansion and contraction causes the gas to perform net work over a cycle (green shaded area). (c) Proposed mechanical autonomous stochastic engine, consisting of two ribbons, main and secondary (displacements $x_{M}$ and $x_{H}$, respectively), and a cantilever (displacement $\left.x_{W}\right)$. (d) Thermal cycle for the proposed engine consisting of four steps: (i) $x_{w}$ is at its leftmost position and energy flows from $x_{H}$ to $x_{M}$, (ii) $M_{w}$ moves to the right $\left(\dot{x}_{w}>0\right)$, while $x_{M}$ stays in a high-energy state, (iii) $x_{w}$ is at its rightmost position, and energy flows from $x_{M}$ to the cold bath, (iv) $x_{w}$ moves back to the initial position while $x_{M}$ stays in a low-energy state.

contact with a cold thermal bath at temperature $T_{C}$ (in our experimental setup $T_{C}$ is the temperature of the ribbon's environment, $293 \mathrm{~K}$ ). The cantilever, also at room temperature, $T_{W}=293 \mathrm{~K}$, acts as a piston that introduces cyclic compressions and expansions of the ribbon and extracts work from the fluctuations in the ribbon's tension. Because of geometric nonlinearity, this tension increases proportionally to the ribbon's vibrational energy and is analogous to the pressure of the gas in a conventional engine. A hot thermal bath, at temperature $T_{H}$, introduces the thermal noise that causes Brownian motion. This heat bath is applied to a secondary ribbon (labeled $x_{H}$ ). The secondary ribbon is weakly coupled to the main ribbon and regulates the coupling between the hot reservoir and the main ribbon [Figs. 1(c) and 1(d)].

Stirling engines require a mechanism to heat and cool the working fluid in synchrony with the motion of the piston. In conventional engines this can be accomplished by a secondary internal piston [Fig. 1(a)] that displaces the fluid, placing it in contact with the hot and cold reservoirs. Prior implementations of the stochastic Stirling cycle used a laser to heat the working particle at predetermined time intervals [6], making the engine nonautonomous. Our engine attains autonomous operation by utilizing the resonance responses of the two ribbons. Because of geometric nonlinearity, the
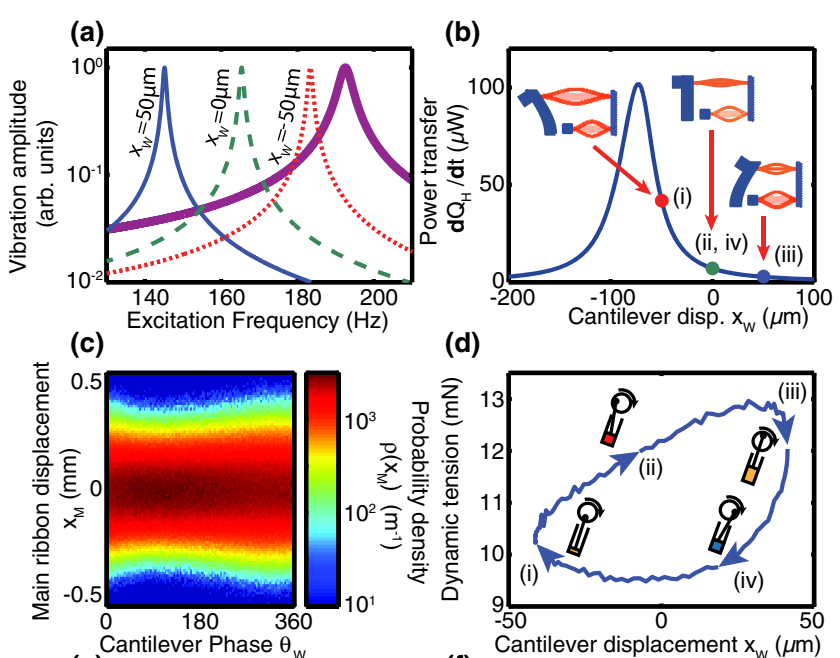

(d)

(e)
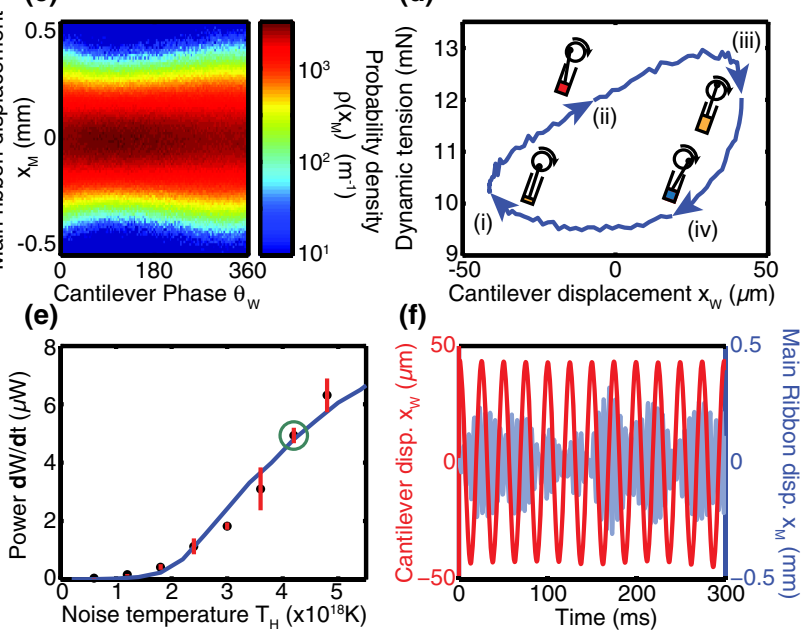

FIG. 2. Thermal engine operation. (a) Theoretical uncoupled frequency response of the main ribbon $\left(x_{M}\right)$, for cantilever displacements $x_{W}$ of $-50 \mu \mathrm{m}$ (blue, solid curve), $0 \mu \mathrm{m}$ (green, dashed curve), and $50 \mu \mathrm{m}$ (red, dotted curve). The uncoupled frequency response of the secondary ribbon $x_{H}$ (thick purple curve) is shown for comparison. (b) Theoretical energy transfer $Q_{H}$ between the hot bath (applied to the secondary ribbon $x_{H}$ ) and the main ribbon $\left(x_{M}\right)$, as a function of the cantilever displacement. The colored dots correspond to the curves in (a). The roman numerals indicate the step of the thermal cycle associated to each displacement and energy transfer. (c) Experimental probability distribution of $x_{M}$ as a function of the cantilever's oscillation phase, $\theta\left(\dot{x}_{W}, x_{W}\right)$. (d) Experimental force acting on the cantilever as a function of the cantilever displacement. (e) Theoretical (blue line) and experimental (black dots) power transfer from the main ribbon to the cantilever as a function of the effective temperature of $x_{H}$. (f) Experimental time evolution of the cantilever (dark red) and ribbon (light blue). The green circle indicates the case $T_{H}=4.2 \times 10^{18} \mathrm{~K}$, corresponding to the experimental conditions depicted in (c), (d), and (f).

resonance frequency of the main ribbon $\left(f_{M}\right)$ depends on the position $x_{W}$ of the cantilever [Fig. 2(a)], while the resonance frequency of the secondary, hot ribbon $\left(f_{H}\right)$ is fixed. As a consequence, the overlap between the respective frequency responses (and therefore the energy transfer [35] between $T_{H}$ and $x_{M}$ ) is controlled by the cantilever [Fig. 2(b)]. By setting the frequency of the main ribbon below the frequency of the secondary ribbon, the maximum energy transfer between the hot bath and the main ribbon occurs when the cantilever is at its leftmost position, as required by the thermal cycle [Fig. 2(b)]. Recent theoretical proposals in optomechanics utilize a similar mechanism to control the coupling between an optical resonator and a heat source [36-38]. 
This tension-mediated feedback mechanism introduces the synchronous heating and cooling required for thermal engine operation without the need of externally prescribed periodic temperature variations as in prior works $[4,6,7]$. The resulting changes in the main ribbon's vibrational energy can be seen in the probability distribution of its position, which is modulated by the cantilever motion [Fig. 2(c)]. The modulation is maximal when the natural frequencies are chosen such that modulation sidebands of the main ribbon's motion coincide with a resonance peak of the coupled system. For weakly coupled ribbons, this condition is approximately $f_{H}-f_{M} \approx f_{W}$. In our experiments, $f_{M}$ varies between 140 and $190 \mathrm{~Hz}, f_{H}=192.55 \mathrm{~Hz}$, and $f_{W}=26.87 \mathrm{~Hz}$ [29].

Figure 2(d) shows the dynamic tension exerted by the main ribbon on the cantilever as a function of the cantilever's position. The dynamic tension was calculated from the measured probability distribution of the ribbon's position $x_{M}$ using the equation $T=\gamma\left\langle x_{M}^{2}\right\rangle$, where $\gamma=513 \mathrm{kN} / \mathrm{m}^{2}$ quantifies the nonlinear coupling between the ribbon's tension and its bending stiffness. This quantity is analogous to the pressure-volume relation in a piston engine. The area inside the curve corresponds to the average work transferred to the cantilever per cycle of operation. This area has a value of $179.2 \pm 7.7 \mathrm{~nJ}$, in good agreement with the average power dissipated in the cantilever, determined to be $171.2 \pm 7.1 \mathrm{~nJ}$ per cycle from the quality factor and average vibrational amplitude. The power output increases nonlinearly with applied noise temperature [Fig. 2(e)]. At $T_{H}=4.2 \times 10^{18} \mathrm{~K}$, its normalized value $P=0.095 \pm 0.009 k_{b} T_{H} \mathrm{~s}^{-1}$ is comparable to that of stochastic engines reported in the literature, whose values are around 0.02 [6] and $5 k_{b} T \mathrm{~s}^{-1}$ [4]. The high effective noise temperatures in Fig. 2(e) are a consequence of the macroscopic dimensions of our tabletop setup, which mandate the use of an external noise excitation. In the Supplemental Material [29], we present simulations on a microscopic engine capable of attaining an output power above $12000 k_{b} T \mathrm{~s}^{-1}$, owing to its high frequency of operation. While our tabletop demonstration requires a significant amount of energy to simulate the high effective temperature $T_{H}$, the microscopic engine does not use any additional energy source besides the heat extracted from $T_{H}$.

During thermal machine operation, the trajectory of the cantilever is approximately a harmonic signal with a slowly varying envelope, while the motion of the ribbon is highly random [Fig. 2(f)]. We further investigate the properties of the cantilever motion by calculating the phase space probability distribution from the experimental measurements [Fig. 3(a)] and theoretical simulations [Fig. 3(b)]. The cantilever's probability distribution is concentrated around a circular orbit, approximating harmonic motion with some amplitude and phase noise (in pure harmonic motion the probability density would be zero everywhere except in a circular, one-dimensional region). This probability distribution is nonpassive: the amount of energy is not minimal given the entropy of the distribution. This (a)

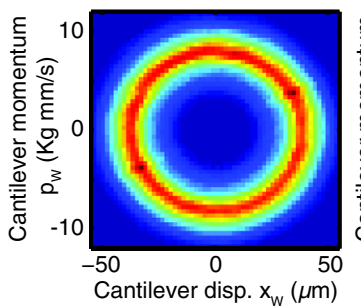

(b)

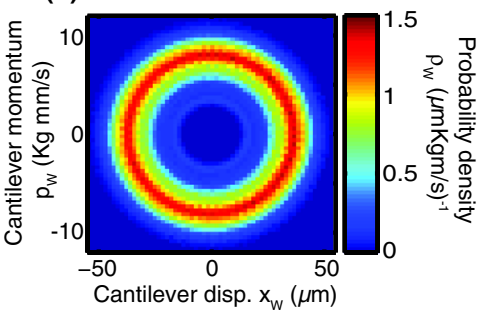

(c)

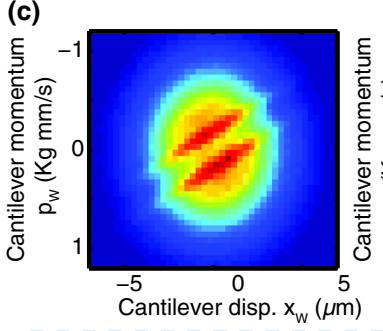

(d)
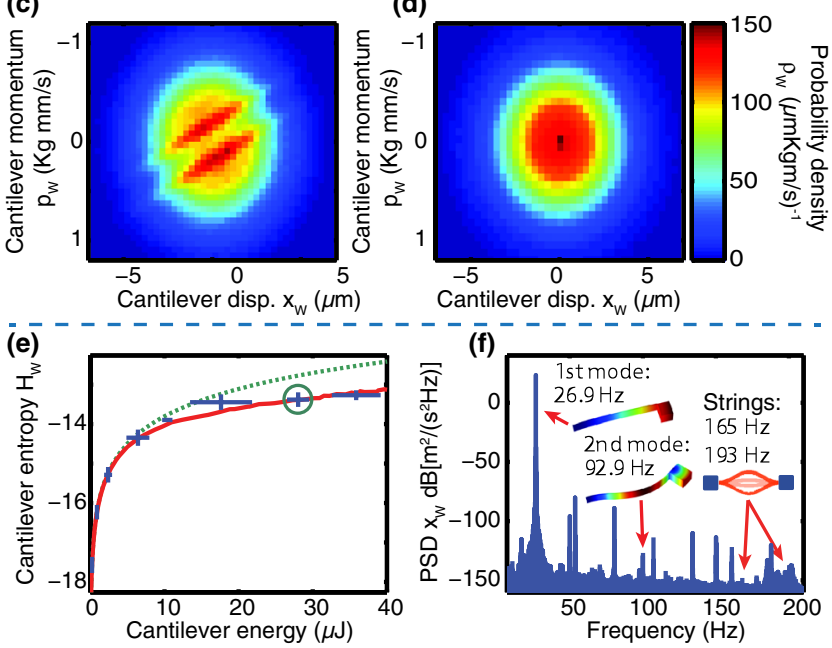

FIG. 3. Properties of the cantilever motion. (a) Experimental phase space probability distribution corresponding to the case when the frequency of the ribbons is tuned to achieve thermal engine operation at $T_{H}=4.2 \times 10^{18} \mathrm{~K}$. (b) Theoretical phase space probability distribution for the experimental case in (a). (c) Phase space probability density function for the cantilever in the detuned system (where $f_{M}>f_{H}$; see Supplemental Material [29] for the exact values) at $T_{H}=2 \times 10^{18} \mathrm{~K}$. (d) Theoretical prediction for the system in (c). (e) Experimental entropy of the cantilever motion as a function of the energy (blue crosses), compared to a theoretical prediction (red line) and to the maximal entropy for the given energy (green dotted line). The green circle indicates the experimental conditions used in (a), (b), and (f), as well as in Figs. 2(c), 2(d), and 2(f). (f) Fourier transform of the experimental cantilever motion.

allows for work extraction without a corresponding flow of entropy [1]. Additionally, the system presents a region of population inversion, with higher probability density around the circular orbit than close to the origin, where the energy is lowest. This distribution is similar to the theoretically predicted Wigner function for a quantum optomechanical heat engine [38], and contrasts with the passive Gaussian distribution describing harmonic oscillators subject to a white noise excitation [39]. We compare the phase space distribution during thermal machine operation [Figs. 3(a) and 3(b)] with that of a detuned system (i.e., where $f_{H}<f_{M}$; see Supplemental Material [29]). In the detuned system, the main ribbon heating is out of sync with the phase of the cantilever oscillation cycle. This prevents thermal machine operation and results in a 
Gaussian phase space probability distribution for the cantilever, maximizing the entropy for a given mean energy [Figs. 3(c) and 3(d)].

We quantify the randomness of the cantilever's motion by calculating the entropy of its phase space probability distribution [Fig. 3(e)]. The difference between the cantilever's entropy and the corresponding equilibrium entropy increases at high cantilever vibrational energies. This indicates the coexistence of two energy transfer mechanisms: an incoherent mechanism analogous to heat transfer [40], where fluctuations of the main ribbon introduce fluctuations on the ribbon's tension that cause the cantilever to move randomly, and a coherent mechanism, where the motion of the main ribbon is modulated by the vibration of the cantilever. At low amplitudes [Fig. 3(e) and Supplemental Material (Animation 1) [29]], or when the main ribbon frequencies are not tuned to result in thermal machine operation [Figs. 3(c) and 3(d)], the incoherent mechanism dominates, resulting in a maximally entropic (passive) probability distribution for the cantilever. At high amplitudes the coherent mechanism becomes significant and the motion of the cantilever is nonpassive, with entropy below the maximal value [Fig. 3(e)]. The Fourier transform of the cantilever velocity [Fig. 3(f)] reveals that its motion occurs mostly at the first mode of resonance. Higher cantilever modes and resonances of the ribbons are below the fundamental component by at least $80 \mathrm{~dB}$. We attribute the presence of small quantities of harmonics to nonlinearities in our measurement system.

The heat engine presented in this Letter corresponds to the mass-spring model in Fig. 4(a) and is described by the system of underdamped [41] Langevin [42,43] equations (see Supplemental Material [29] for derivation and numerical algorithms):

$$
\begin{aligned}
& m_{H} \ddot{x}_{H}+b_{H} \dot{x}_{H}+k_{H} x_{H}+k_{H M}\left(x_{H}-x_{M}\right)+\mu x_{H}^{3}=\xi_{H}, \\
& m_{M} \ddot{x}_{M}+b_{M} \dot{x}_{M}+\left(k_{M}-2 \gamma x_{W}\right) x_{M} \\
& \quad+k_{H M}\left(x_{M}-x_{H}\right)+\mu x_{M}^{3}=\xi_{C}, \\
& m_{W} \ddot{x}_{W}+b_{W} \dot{x}_{W}+k_{W} x_{W}-\gamma x_{M}^{2}=\xi_{W} .
\end{aligned}
$$

Here, $k_{H}, k_{M}$, and $k_{W}$ are the stiffness of the hot ribbon, the main ribbon, and the cantilever, respectively, $b_{H}, b_{M}$, and $b_{W}$ are the corresponding linear damping coefficients, and $m_{H}, m_{M}$, and $m_{W}$ are the corresponding masses. The terms $\xi_{H}, \xi_{C}$, and $\xi_{W}$ represent the thermal noise introduced by the baths acting on each degree of freedom. These terms have a white noise power spectral density of $4 K_{B} T_{X} b_{X}$ [44]. This relationship between dissipation, temperature, and excitation force is used to determine the experimental effective temperature. In the numerical simulations, the temperature $T_{H}$ is set to match the excitation used in the experiments, while $T_{C}$ and $T_{W}$ are set to zero (except otherwise indicated), since they are negligible in comparison to $T_{H}$. The constant $k_{H M}$ represents the linear coupling between the two ribbons, $\gamma$ is the nonlinear coupling between the main ribbon and the cantilever (also appearing in the ribbon's dynamic tension equation), and $\mu$ is the cubic nonlinear stiffness of the ribbons. Experimentally measured values for the all parameters are provided in the Supplemental Material [29]. Coupled degrees of freedom subject to Brownian motion have been studied in electronic systems [40], and the asymmetric coupling between $x_{M}$ and $x_{W}$ appears in the description of phonon modes in superconductors [45].

As demonstrated in Fig. 3, the theoretical model predictions are in good agreement with the experiments. Thus, we use the model to determine quantities that are not directly measurable in our experimental setup, such as the energy transfer between ribbons. We highlight the most relevant theoretical findings in Fig. 4. We define the power transfer between a degree of freedom and its thermal bath as the work of the thermal noise and damping: $\boldsymbol{d} W_{X} / \boldsymbol{d} t=$ $\left\langle\left(b_{X} \dot{x}_{X}-\xi_{X}\right) \dot{x}_{X}\right\rangle$ [46]. We first study the effect of the cantilever thermal bath's temperature $T_{W}$. Increasing $T_{W}$ increases the energy transferred between $T_{H}$ and $T_{W}$ [Fig. 4(a)]. This corresponds to an effective negative thermal conductivity. In addition, the system is able to transfer energy between the thermal baths $T_{H}$ and $T_{W}$ even when $T_{W}$ is increased above $T_{H}$. This observation, which seems to defy the second law of thermodynamics, is made possible by the fact that $x_{M}$ is at a lower temperature than $x_{H}$ and absorbs the excess entropy extracted from $\xi_{H}$. Figure 4(c) presents the
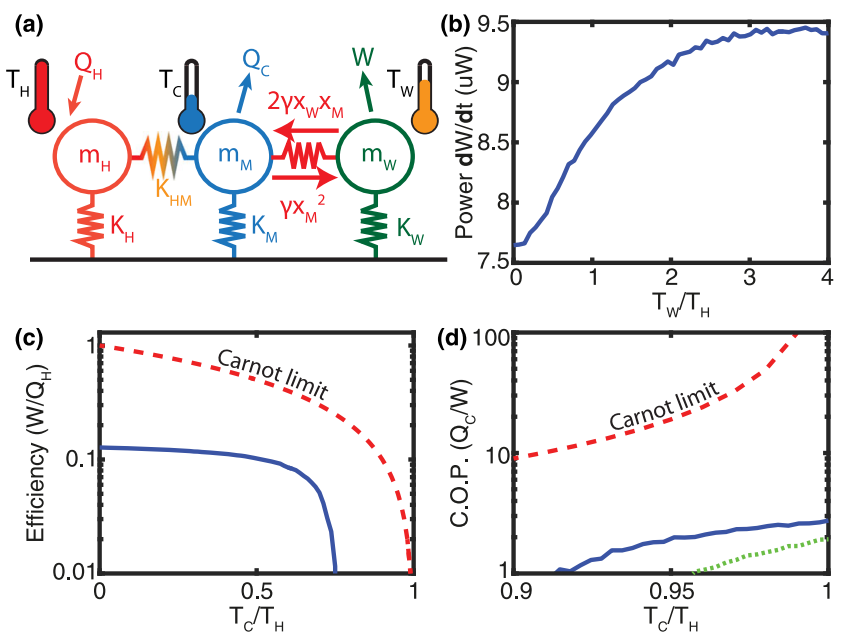

FIG. 4. Theoretical investigation. (a) Mass-spring model for the system. (b) Energy transfer as a function of the cantilever temperature $T_{W}$. (c) Efficiency of the thermal machine (blue, solid line) and comparison with the Carnot efficiency (red, dashed line). Here, the cantilever motion has been prescribed to be $x_{W}=(50 \mu \mathrm{m}) \cos \left(\omega_{W} t\right)$ to prevent incoherent energy transfer between the ribbons and the cantilever. (d) Refrigerator coefficient of performance (C.O.P. $=Q_{C} / W$ ) when the cantilever is forced to oscillate at $A_{W}=50 \mu \mathrm{m}$ (blue, solid line) and when driven by noise at $T_{W}=k_{W} A_{W}^{2} / k_{B}$ (green, dotted line). The red dashed line is the Carnot maximal C.O.P. 
efficiency of the thermal machine as a function of the ratio between $T_{H}$ and $T_{C}$. The machine attains a maximum efficiency of approximately $30 \%$ of the maximal Carnot efficiency using our experimental, unoptimized parameters. In the Supplemental Material [29] we present alternative designs that attain efficiencies up to $50 \%$ of the theoretical maximum. When the temperature ratio $T_{H} / T_{C}$ is close to 1 , the energy flow between $T_{H}$ and $T_{C}$ reverses, and the machine acts as a refrigerator [23]. The refrigerator regime requires a constant supply of energy to the cantilever. This energy can be provided by externally prescribing the cantilever displacement, or by increasing $T_{W}$ to introduce large amplitude thermal motion in the cantilever. In the latter case, the main ribbon is cooled through the addition of heat to the system, which behaves as an absorption refrigerator [36,47-50]. Figure 4(d) presents the efficiency of the refrigerator operation.

This work has demonstrated that a mechanical system consisting of two ribbons and a cantilever has the ability to act as a heat engine or refrigerator, and presents the unusual property of negative thermal conductivity. Traditionally, Brownian motion has been seen as an inconvenience when present in mechanical systems, e.g., by limiting the precision of nanomechanical sensors [51]. Our work demonstrates that this thermal noise may be a source of energy and a tool to study thermodynamics in both macroand microscale systems. This work was supported by ETH Research Grant No. ETH-24 15-2 and ETH Zurich Foundation Seed Project ESC-A 06-14.

*sermarc@ethz.ch

[1] D. Gelbwaser-Klimovsky and G. Kurizki, Work extraction from heat-powered quantized optomechanical setups, Sci. Rep. 5, 7809 (2015).

[2] T. Komatsu, N. Nakagawa, S.-i. Sasa, and H. Tasaki, Exact equalities and thermodynamic relations for nonequilibrium steady states, J. Stat. Phys. 159, 1237 (2015).

[3] U. Seifert, Stochastic thermodynamics, fluctuation theorems and molecular machines, Rep. Prog. Phys. 75, 126001 (2012).

[4] I. A. Martinez, E. Roldan, L. Dinis, D. Petrov, J. M. R. Parrondo, and R. A. Rica, Brownian Carnot engine, Nat. Phys. 12, 67 (2016).

[5] S. Toyabe, T. Sagawa, M. Ueda, E. Muneyuki, and M. Sano, Experimental demonstration of information-to-energy conversion and validation of the generalized Jarzynski equality, Nat. Phys. 6, 988 (2010).

[6] V. Blickle and C. Bechinger, Realization of a micrometresized stochastic heat engine, Nat. Phys. 8, 143 (2012).

[7] I. A. Martínez, É. Roldán, L. Dinis, D. Petrov, and R. A. Rica, Adiabatic Processes Realized with a Trapped Brownian Particle, Phys. Rev. Lett. 114, 120601 (2015).

[8] J. V. Koski, A. Kutvonen, I. M. Khaymovich, T. Ala-Nissila, and J.P. Pekola, On-Chip Maxwell's Demon as an Information-Powered Refrigerator, Phys. Rev. Lett. 115, 260602 (2015).
[9] D. Mandal and C. Jarzynski, Work and information processing in a solvable model of Maxwell's demon, Proc. Natl. Acad. Sci. U.S.A. 109, 11641 (2012).

[10] P. Strasberg, G. Schaller, T. Brandes, and M. Esposito, Thermodynamics of a Physical Model Implementing a Maxwell Demon, Phys. Rev. Lett. 110, 040601 (2013).

[11] D. M. Zhiyue Lu and J. Christopher, Engineering Maxwell's demon, Phys. Today 67, No. 8, 60 (2014).

[12] P. Strasberg, G. Schaller, T. Brandes, and C. Jarzynski, Second laws for an information driven current through a spin valve, Phys. Rev. E 90, 062107 (2014).

[13] C. Jarzynski, Nonequilibrium Equality for Free Energy Differences, Phys. Rev. Lett. 78, 2690 (1997).

[14] U. Seifert, Entropy Production along a Stochastic Trajectory and an Integral Fluctuation Theorem, Phys. Rev. Lett. 95, 040602 (2005).

[15] Y. Utsumi and T. Taniguchi, Fluctuation Theorem for a Small Engine and Magnetization Switching by Spin Torque, Phys. Rev. Lett. 114, 186601 (2015).

[16] G. E. Crooks, Entropy production fluctuation theorem and the nonequilibrium work relation for free energy differences, Phys. Rev. E 60, 2721 (1999).

[17] J. M. Horowitz and M. Esposito, Thermodynamics with Continuous Information Flow, Phys. Rev. X 4, 031015 (2014).

[18] C. Bennett, The thermodynamics of computation-A review, Int. J. Theor. Phys. 21, 905 (1982).

[19] J. Hoppenau and A. Engel, On the energetics of information exchange, Europhys. Lett. 105, 50002 (2014).

[20] J. M. R. Parrondo, J. M. Horowitz, and T. Sagawa, Thermodynamics of information, Nat. Phys. 11, 131 (2015).

[21] H. Viktor, An exactly solvable model of a stochastic heat engine: Optimization of power, power fluctuations and efficiency, J. Stat. Mech. (2015) P05022.

[22] T. Schmiedl and U. Seifert, Efficiency at maximum power: An analytically solvable model for stochastic heat engines, Europhys. Lett. 81, 20003 (2008).

[23] M. Esposito, N. Kumar, K. Lindenberg, and C. Van den Broeck, Stochastically driven single-level quantum dot: A nanoscale finite-time thermodynamic machine and its various operational modes, Phys. Rev. E 85, 031117 (2012).

[24] W. Pusz and S. L. Woronowicz, Passive states and KMS states for general quantum systems, Commun. Math. Phys. 58, 273 (1978).

[25] A. Lenard, Thermodynamical proof of the Gibbs formula for elementary quantum systems, J. Stat. Phys. 19, 575 (1978).

[26] J. C. Maxwell, Theory of Heat (Longmans Green, and Co, London, 1871).

[27] M. v. Smoluchowski, Experimentally verifiable molecular phenomena that contradict ordinary thermodynamics, Phys. Z. 8, 1069 (1912).

[28] R. P. Feynman, R. B. Leighton, and M. Sands, The Feynman Lectures on Physics I (Addison-Wesley, Reading, MA, 1963).

[29] See Supplemental Material at http://link.aps.org/ supplemental/10.1103/PhysRevLett.117.010602, which includes Refs. [30-34], contains alternative heat engine designs, additional details on the experimental system, a description of the numerical and experimental methods, and a theoretical study on the scaling of the engine to microscopic dimensions. 
[30] S. Woinowski-Krieger, The effect of an axial force on the vibration of hinged bars, J. Appl. Mech. 17, 35 (1950).

[31] H. W. C. Postma, I. Kozinsky, A. Husain, and M. L. Roukes, Dynamic range of nanotube- and nanowire-based electromechanical systems, Appl. Phys. Lett. 86, 223105 (2005).

[32] A. Rößler, Runge-Kutta methods for the strong approximation of solutions of stochastic differential equations, SIAM J. Numer. Anal. 48, 922 (2010).

[33] M. J. Burek, D. Ramos, P. Patel, I. W. Frank, and M. Lončar, Nanomechanical resonant structures in single-crystal diamond, Appl. Phys. Lett. 103, 131904 (2013).

[34] D. Garcia-Sanchez, A. M. van der Zande, A. S. Paulo, B. Lassagne, P. L. McEuen, and A. Bachtold, Imaging mechanical vibrations in suspended graphene sheets, Nano Lett. 8, 1399 (2008).

[35] D. E. Newland, Calculation of power flow between coupled oscillators, J. Sound Vib. 3, 262 (1966).

[36] A. Mari and J. Eisert, Cooling by Heating: Very Hot Thermal Light Can Significantly Cool Quantum Systems, Phys. Rev. Lett. 108, 120602 (2012).

[37] K. Zhang, F. Bariani, and P. Meystre, Quantum Optomechanical Heat Engine, Phys. Rev. Lett. 112, 150602 (2014).

[38] A. Mari, A. Farace, and V. Giovannetti, Quantum optomechanical piston engines powered by heat, J. Phys. B 48, 175501 (2015).

[39] W. Ebeling and I. Sokolov, Statistical Thermodynamics and Stochastic Theory of Nonequilibrium Systems (World Scientific, Singapore, 2005).

[40] S. Ciliberto, A. Imparato, A. Naert, and M. Tanase, Heat Flux and Entropy Produced by Thermal Fluctuations, Phys. Rev. Lett. 110, 180601 (2013).
[41] M. S. Simon, J. M. Sancho, and K. Lindenberg, Transport and diffusion of underdamped Brownian particles in random potentials, Eur. Phys. J. B 87, 10 (2014).

[42] P. Langevin, Sur la theorie du mouvement Brownien, C.R. Hebd. Seances Acad. Sci. 146, 530 (1908); G. E. Uhlenbeck and L. S. Ornstein, On the theory of the Brownian motion, Phys. Rev. 36, 823 (1930)

[43] D. S. Lemons and A. Gythiel, Paul Langevin's 1908 paper "On the theory of Brownian motion", Am. J. Phys. 65, 1079 (1997).

[44] K. Sekimoto, Stochastic Energetics (Springer-Verlag, Heidelberg, 2010).

[45] R. Mankowsky, A. Subedi, M. Forst, S. O. Mariager, M. Chollet, H. T. Lemke et al., Nonlinear lattice dynamics as a basis for enhanced superconductivity in $\mathrm{YBa}_{2} \mathrm{Cu}_{3} \mathrm{O}_{6.5}$, Nature (London) 516, 71 (2014).

[46] K. Sekimoto, Langevin equation and thermodynamics, Prog. Theor. Phys. Suppl. 130, 17 (1998).

[47] N. Linden, S. Popescu, and P. Skrzypczyk, How Small Can Thermal Machines Be? The Smallest Possible Refrigerator, Phys. Rev. Lett. 105, 130401 (2010).

[48] A. Levy and R. Kosloff, Quantum Absorption Refrigerator, Phys. Rev. Lett. 108, 070604 (2012).

[49] L. A. Correa, J. P. Palao, D. Alonso, and G. Adesso, Quantum-enhanced absorption refrigerators, Sci. Rep. 4, 3949 (2014).

[50] R. Silva, P. Skrzypczyk, and N. Brunner, Small quantum absorption refrigerator with reversed couplings, Phys. Rev. E 92, 012136 (2015).

[51] K. L. Ekinci, Y. T. Yang, and M. L. Roukes, Ultimate limits to inertial mass sensing based upon nanoelectromechanical systems, J. Appl. Phys. 95, 2682 (2004). 